and water transportation in China, and has the name of "nine province main thoroughfares."

In 2000, Wuhan Medical Emergency Center and Wuhan Helicopter General Aviation Co. Ltd signed an agreement to make use of two Enstrom 480 helicopters to provide aerial rescue services. The aero radius is $180 \mathrm{~km}$, or 11 flight hours. The helicopter rescue expert team consists of 65 high-grade doctors from 16 professional subjects of 15 large hospitals. We already have transacted the " $\mathrm{A}$ " flight permission procedure; we can fly as soon as we get the dispatch report, weather permitting. So far, the service has rescued five critically ill patients.

At the present time, Wuhan is the only large city in which the citizens can dial " 120 " to dispatch rescue helicopters. The EMS system shows the solid construction of aquatic, overland, and aerial rescue.

Keywords: emergency medical services (EMS); helicopter; rescue; system Prebosp Disast Med 2002;17(s2):s85-s86.

E-mail: wh120@vip.sina.com

\section{Chlorine Gas Lung Injury: Intravenous Versus Aerosolized Corticosteroid Treatment in a Porcine Model}

Jianpu Wang, MD; Liming Zhang, MD; Sten $M$. Walther, $M D, P h D$

Objective: Chlorine gas remains one of the most common gaseous intoxicants due to its widespread use in modern society. Current treatment of acute chlorine gas injury is mainly symptomatic. This blind placebo-controlled study examined the effects of post-injury corticosteroid administration in chlorine gas injured pigs followed for 24 hours. Method: Anesthetized and mechanically ventilated pigs were exposed to chlorine gas ( $400 \mathrm{ppm})$ for 15 minutes, then assigned to three groups: 30 minutes after gassing each group received either aerosolized budesonide $(n=8)$, intravenous betamethasone $(n=8)$, or placebo (doubledummy, $n=8$ ). Hemodynamics, gas exchange, and lung mechanics were evaluated for 24 hours.

Results: Airway and pulmonary artery pressure increased, and arterial oxygenation fell precipitously in all animals (from $13.5 \pm 0.8$ to $6.5 \pm 0.9 \mathrm{kPa}, p<0.001$ ). These immediate changes were followed by a gradual improvement over six to eight hours to a stable level of dysfunction for the remainder of the experiment in placebo animals. Arterial oxygen tension, pulmonary vascular resistance, and airway pressure recovered faster and more completely in the budesonide and betamethasone groups than in the placebo group $(p<0.01)$. Lung wet to dry weight ratio was greater in the placebo group than in the budesonide and betamethasone groups ( $6.34 \pm 0.59$ vs. $5.56 \pm 0.38$ and 5.53 \pm 0.54 , respectively. $p<0.05$ ).

Conclusion: Treatment of chlorine gas lung injury with aerosolized budesonide or intravenous betamethasone improved symptoms and signs of lung injury compared with placebo. This study supports early treatment with corticosteroids in victims of chlorine gas injury.

Keywords: chlorine gas; corticosteroids; lung injury Prehosp Disast Med 2002;17(s2):s86.

\section{Evaluation of Disastrous Consequences of BSE}

Epidemic with Public Panic in Japan

Shinichiro Yanagisawa, MCS; ${ }^{1}$ Ko Nakajo, $M S,{ }^{2}$ Hiroshi

Aino, $M S_{;}^{2}$ Hiroki Inoue, $M D, D M D,{ }^{2} L i C a i, M D,{ }^{2}$

Isao Kamae, $M D, D r P H^{1,2}$

1. Research Center for Urban Safety and Security, Kobe University

2. Kobe University School of Medicine

Background: In 2001, the detection of bovine spongiform encephalopathy (BSE) in Japan, the first case in Asia, made the public extremely nervous about the safety of food products containing beef, which led to deteriorating consequences on the beef market in Japan. Unfortunately, the anthrax threats in the United States in the same year drove the BSE epidemic to a kind of "media-induced" disaster in the society.

Objective: To address the public concern over the BSE epidemic, the mass screening system established by the Japanese government was reviewed with respect to its advantages and limitations.

Methods: Based on the evidence from BSE tests reported by the European Union, the accuracy of the screening system in Japan was investigated, and the number of false negatives and positives under the current system was estimated. Results: The analysis indicated that, since Japanese cattle were still at low risk, there could exist a very small quantity of error in BSE detection with less than one false negative in four years. However, it was confirmed that the current system is far from the best for protecting humans from the BSE hazard.

Conclusion: Despite the BSE epidemic, the human risk for BSE in Japan is quite low in Japan compared with other medical risks. Scientifically rational communication through the media will be needed to prevent the next mass panic caused by irrationality.

Keywords: bovine spongiform encephalopathy (BSE); epidemic; media; public concern; risk; screening

Prebosp Disast Med 2002;17(s2):s86.

\section{Enhancement of Management for Land \\ Transportation of Dangerous Chemicals Zhu Mingxue; Zhao Jie}

Department of Naval Medicine, Second Military Medical University, Shanghai, People's Republic of China

Every day, there are many vehicles transporting dangerous chemicals on the expressways. If the chemicals leak, catch on fire, or explode, a serious chemical event would involve the dense population living along the way.

1. Management issues associated with transportation of dangerous chemicals

a. Transportation without proper certificates or with disqualification certificates

b. Driving not in accord with the time scheme and designed traffic line

c. Imperfect safety regulations for blocking leaks and fire control

d. Drivers and escorts working without training

2. Reasons and lessons from chemical accidents

a. Some special vehicles transporting dangerous chemicals aren't qualified by the correlative governmental administration 